\title{
Signature of quantum criticality in photoemission spectroscopy
}

\author{
M. Klein, ${ }^{1}$ A. Nuber,${ }^{1}$ F. Reinert,${ }^{1,2}$ J. Kroha,${ }^{3}$ O. Stockert,${ }^{4}$ and H. v. Löhneysen ${ }^{5,6}$ \\ ${ }^{1}$ Universität Würzburg, Experimentelle Physik II, Am Hubland, D-97074 Würzburg, Germany \\ ${ }^{2}$ Forschungszentrum Karlsruhe, Gemeinschaftslabor für Nanoanalytik, D-76021 Karlsruhe, Germany \\ ${ }^{3}$ Universität Bonn, Physikalisches Institut, Nussallee 12, D-53115 Bonn, Germany \\ ${ }^{4}$ Max Planck Institute for Chemical Physics of Solids, Nöthnitzer Str. 40, 01187 Dresden, Germany \\ ${ }^{5}$ Physikalisches Institut, Universität Karlsruhe (TH), D-76128 Karlsruhe, Germany \\ ${ }^{6}$ Forschungszentrum Karlsruhe, Institut für Festköperphysik, D-76021 Karlsruhe, Germany
}

(Dated: March 7, 2022)

\begin{abstract}
A quantum phase transition (QPT) in a heavy-fermion (HF) compound may destroy the Fermi liquid groundstate. However, the conditions for this breakdown have remained obscure. We report the first direct investigation of heavy quasiparticle formation and breakdown in the canonical system $\mathrm{CeCu}_{6-x} \mathrm{Au}_{x}$ by ultraviolet photoemission spectroscopy at elevated temperatures without the complications of lattice coherence. Surprisingly, the single-ion Kondo energy scale $T_{K}$ exhibits an abrupt step near the quantum critical Au concentration of $x_{c}=0.1$. We show theoretically that this step is expected from a highly non-linear renormalization of the local spin coupling at each Ce site, induced by spin fluctuations on neighboring sites. It provides a general high-temperature indicator for HF quasiparticle breakdown at a QPT.
\end{abstract}

PACS numbers: 71.27.+a 71.28.+d 79.60.-i 71.10.-w

A quantum phase transition (QPT) is a second-order transition occurring at zero temperature, driven by a nonthermal control parameter such as composition or pressure. Here, two competing groundstates are separated by a quantum critical point (QCP). Heavy-fermion (HF) systems, in particular, show striking deviations from Fermi-liquid (FL) behavior at a QPC between a magnetically ordered and a paramagnetic phase [1, 2]. In the standard Hertz-Millis (HM) scenario [3, 4] only the bosonic fluctuations of the order parameter become quantum critical, i.e., long-ranged in space and time, leading to anomalous behavior of physical quantities, but leaving the fermionic quasiparticles intact. However, in a heavy fermion (HF) system the order-parameter fluctuations always couple to the spin degree of freedom of the fermionic excitations, so that the latter may become critical as well and disintegrate. In this case, the formation of the Kondo spin singlet between the conduction electron and the local $4 f$ magnetic moments is prevented, and hence the Kondo scale $T_{K}$ vanishes at the quantum critical point (QCP). This scenario has, therefore, been termed local quantum critical (LQC) [5, [6] $\mathrm{CeCu}_{6-x} \mathrm{Au}_{x}$ is one of the best characterized HF compounds 7, , $8,9,10,11,12,13$ with a QPT between a fully Kondo-screened paramagnetic and an antiferromagnetically ordered phase at a critical Au concentration of $x_{c}=0.1$. Based on inelastic neutron scattering experiments it was suggested that the LQC scenario is realized in this compound [14], yet this issue has remained controversial.

In order to address the crucial question whether Kondo screening persists or not at the QPT, we present in this work ultraviolet photoemission spectroscopy (UPS) measurements, which provide the most immediate access to the screening scale $T_{K}$ by directly recording the Kondo resonance $(\mathrm{KR})$ in the local Ce $4 f$ spectrum [15, 16].
By performing the experiments at temperatures $T$ well above the Neél temperature and above the lattice coherence temperature $\left(T>\left\{T_{K}, T_{c o h} ; T_{N}\right\}\right)$ we are able to investigate the local spin screening effect alone, without the complications of Fermi-volume change due to lattice coherence, which influence the results of other experimental techniques.

The high-resolution UPS was performed with a Gammadata R4000 analyzer and a monochromatized VUVlamp at $h \nu=40.8 \mathrm{eV}$ (see Refs. [16] and [17] for more details). We cleaved the single crystalline samples in situ just before the measurement, already at the measurement temperature. The surface is known to be $\gamma$-Ce-like, meaning that the surface properties do not differ from the bulk properties 16 .

The inset of Fig. 1a) shows survey spectra for five $\mathrm{Au}$ concentrations $x$ at $T=15 \mathrm{~K}$. The energy range includes the spin-orbit (SO) feature at $E_{B} \approx 260 \mathrm{meV}$ and the tail of the Kondo resonance just below the Fermi energy $E_{F}$. A distinct crystal-field (CF) feature as observed, e.g., in $\mathrm{CeCu}_{2} \mathrm{Si}_{2}$ and $\mathrm{CeNi}_{2} \mathrm{Ge}_{2}[15,16]$, is expected near $E_{B}=7 \mathrm{meV}$, but strongly broadened [12] and, hence, not discernible in $\mathrm{CeCu}_{6-x} \mathrm{Au}_{x}$. The $\mathrm{SO}$ peak does not show a significant $x$-dependent variation in position or intensity. This is expected, since it corresponds to an only virtually excited initial state of the UPS process [15, 18]. In order to investigate the KR in more detail we performed high-resolution measurements near $E_{F}$ (Fig. 1a)). For all concentrations one observes a significant rise in the intensity from $E_{B} \approx 20 \mathrm{meV}$ towards $E_{F}$, attributed to the tail of the $\mathrm{KR}$ which has its major spectral weight a few meV above the Fermi energy [15, 16] suppressed by the Fermi-Dirac distribution function (FDD) in the UPS data. Normalizing to the FDD allows to recover the thermally occupied spectrum up to $\approx 5 k_{B} T$ above 

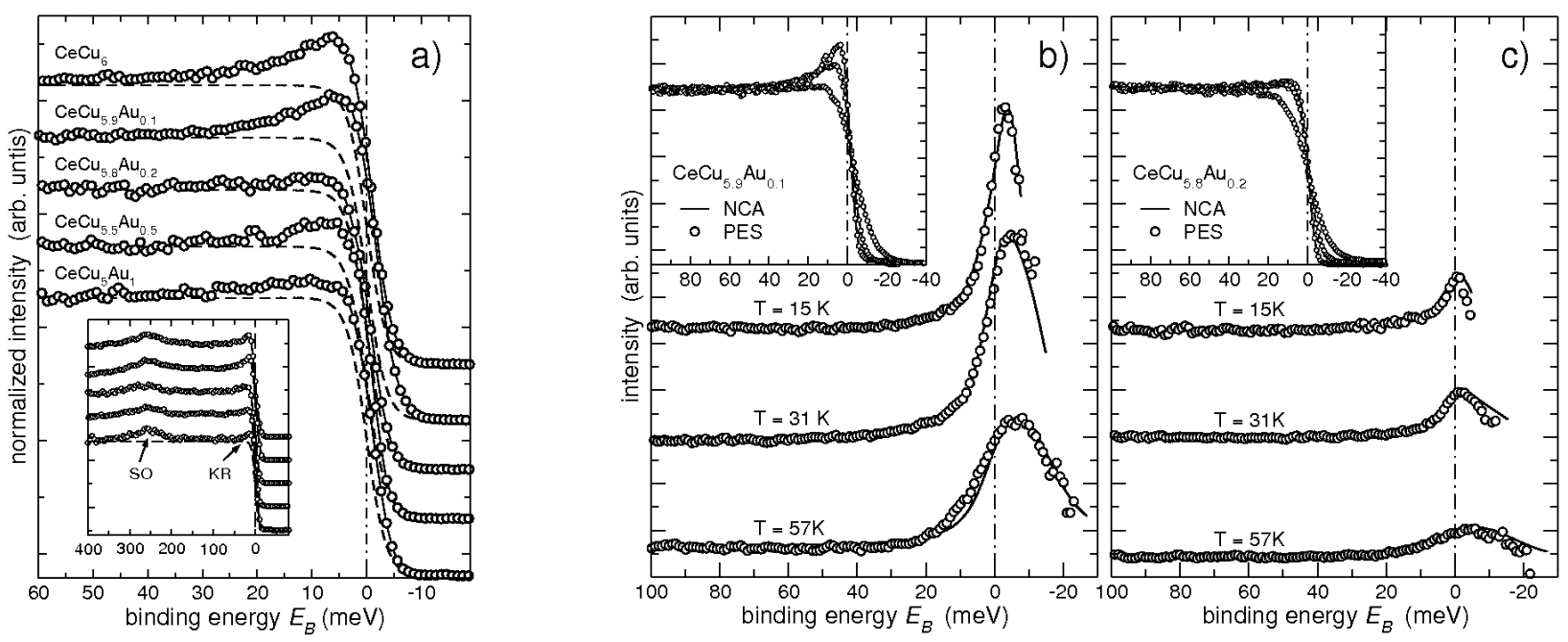

FIG. 1: a) Near- $E_{F}$ spectra of $\mathrm{CeCu}_{6-x} \mathrm{Au}_{x}$ for five different $\mathrm{Au}$ concentrations at $T=15 \mathrm{~K}(h \nu=40.8 \mathrm{eVn} \Delta E=4.9 \mathrm{meV})$, normalized at $E_{B} \approx 100 \mathrm{meV}$. The dashed lines describe the resolution broadened FDD at $T=15 \mathrm{~K}$. The inset shows a larger energy range including the spin-orbit (SO) partner at $\left.E_{B} \approx 260 \mathrm{meV}(\Delta E=15 \mathrm{meV})\right)$. b) and c) show spectra for $x=0.1$ and $x=0.2$, respectively, devided by the FDD, at various $T$. The solid lines are best NCA fits (see Tab. I for the resulting model parameter values). The insets in b) and c) show the corresponding raw data.

$E_{F}[15,16]$ and reveals the KR. Fig. 1 1 shows the FDDrenormalized spectra for $x=0.1(\mathrm{~b}))$ and $0.2(\mathrm{c})$ ). In the low- $T$ spectra $(T=15 \mathrm{~K})$ its maximum is at about $3 \mathrm{meV}$ above $E_{F}$ for $x=0.1$ and at $1 \mathrm{meV}$ for $x=0.2$. The striking feature seen in Figs. (1b) and c) is the significant drop of the spectral weight of the KR from $x=0.1$ to 0.2 , i.e., in the vicinity of the critical concentration $x_{c}$. The differences in the shape and the $T$ dependence between the $x=0$ and 0.1 spectra as well as the differences between the $x=0.2,0.5$, and 1.0 spectra (not shown) are negligibly small compared to the jump close to $x_{c}$.

To extract the Kondo screening scale $T_{K}$ from the experimental results, one must bear in mind that the coherence temperature $T_{c o h}$ obtained from resistivity measurements 19] as well as the Kondo scale extracted from specific heat 20] or neutron scattering data 12, 14], are well below our lowest experimental temperature. Moreover, quantum critical fluctuations, which in $\mathrm{CeCu}_{6-x} \mathrm{Au}_{x}$ extend up to about $T=7 \mathrm{~K}$ [14] and certainly become crucial at low $T$, are not expected to influence our exper-

\begin{tabular}{cccc}
$x$ & $\Delta_{C F 1,2}(\mathrm{meV})$ & $V(\mathrm{meV})$ & $T_{K}(\mathrm{~K})$ \\
\hline 0 & $7.2,13.9$ & 116 & 4.6 \\
0.1 & $7.2,13.9$ & 116 & 4.6 \\
0.2 & $8.7,13.6$ & 111 & 3.4 \\
0.5 & $8.9,13.6$ & 109 & 3.3 \\
1 & $9.2,13.6$ & 108 & 3.1
\end{tabular}

TABLE I: SIAM/NCA fit parameters, CF splittings of the Ce $4 f$ orbitals, $\Delta_{C F 1}, \Delta_{C F 2}, 4 f$-conduction band hybridization matrix element, $V$, and the resulting Kondo temperatures, $T_{K}$, for different $\mathrm{Au}$ concentrations $x$. Fixed parameters: conduction-band half-width $D_{0}=2.8 \mathrm{eV}$ (HWHM), single-particle $4 f$ binding energy $\varepsilon_{f}=-1.05 \mathrm{eV}$, SO splitting $\Delta_{S O}=250 \mathrm{meV}$. imental spectra. Thus, our data exhibit the onset of the local Kondo physics on the Ce atoms only. Therefore, the single-impurity Anderson model (SIAM) is employed to interpret the experimental data. To determine $T_{K}$ we follow the procedure successfully applied to various $\mathrm{Ce}$ compounds in the past [15, 16, 21, 22, 23, 24]: Using the noncrossing approximation (NCA) [25] we calculate the Ce $4 f$ spectral function of the SIAM, including all $\mathrm{CF}$ and SO excitations. For each composition $x$ the NCA spectra are broadened by the experimental resulotion and fitted to the experimental data, using a single parameter set for all experimental $T$. The NCA spectra are then computationally extrapolated to $T \approx 0.1 T_{K}$, where $T_{K}$ is extracted from the Kondo-peak half-width at half maximum (HWHM) of the NCA spectra. The resulting fit parameter values are shown in Tab. I. Fig. 2 shows the $x$ dependence of $T_{K}$ for $\mathrm{CeCu}_{6-x} \mathrm{Au}_{x}$ obtained from our UPS data, compared to results of various other experiments [12, 19, 20]. We emphasize that, irrespective of a possible systematic ambiguity in the fit procedure, the surprisingly abrupt step of $T_{K}$ near $x=x_{c} \approx 0.1$ is significant and already clearly visible in the raw data (Fig. (1).

What can be learnt from this step-like behavior at elevated $T$ about the nature of the QPT at $T=0 \mathrm{~K}$ ? The method of extracting a Kondo scale from a singleion picture described above mimics exactly a system of dense Kondo atoms that, however, would not undergo any lattice coherence or magnetic ordering at sufficiently low $T$. This scenario is different from the low- $T$ behavior of the Kondo lattice and two-impurity Kondo problems, where Kondo spin screnning would have to compete with the formation of inter-impurity coherent states [26] or could be destroyed by critical fluctuations of a magnetic order parameter [27]. By contrast, our sys- 


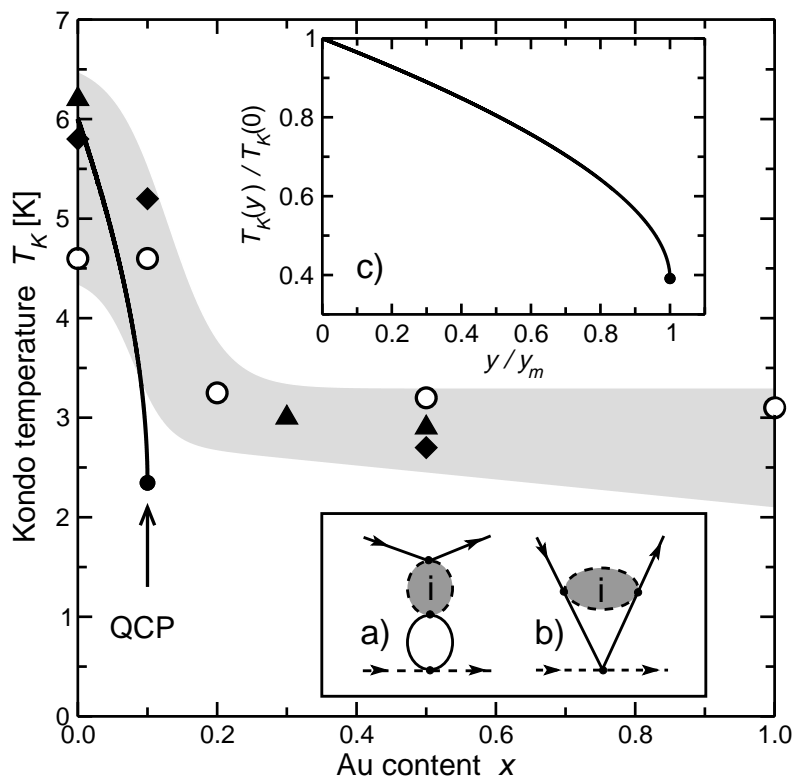

FIG. 2: Dependence of the Kondo temperature $T_{K}$ on the $\mathrm{Au}$ content $x$, as determined by UPS (open circles), specific heat [20] (triangles) and neutron scattering [12, 14] (diamonds). The shaded area is a guide to the eye. The insets a) and b) show diagrammatic representations of the RKKY corrections to the local Kondo vertex. The inset c) and the solid line in the main panel show the universal curve $T_{K}(y) / T_{K}(0)$ vs. $y / y_{m}$ as given by Eq. (3).

tem is represented by an effective single-impurity Kondo model where the Kondo exchange coupling $J$ is renormalized by the local spin fluctuations on the surrounding identical Kondo atoms through the indirect RudermanKittel-Kasuya-Yosida (RKKY) coupling. This problem can be treated in a controlled way by renormalization group (RG) enhanced perturbation theory which allows for definite predictions about the formation of HF quasiparticles at low $T$, as we will show in the following.

The leading perturbative corrections to $J$ on a given Ce atom at lattice site $i=0$ are depicted diagrammatically in the insets a), b) of Fig. 2. They obviously involve the full dynamical spin susceptibility $\chi_{4 f}(T, \Omega)$ of a local Ce $4 f$ spin on a neighboring site $i \neq 0$, where $\chi_{4 f}(T, 0)=$ $\left(g_{L} \mu_{B}\right)^{2} N(0) D_{0} /\left(4 \sqrt{T_{K}^{2}+T^{2}}\right)$, with $N(0)$ the density of states at the Fermi level, $D_{0} \approx E_{F}$ the band cutoff and $g_{L}, \mu_{B}$ the Landé factor and the Bohr magneton, respectively. Summing over all lattice sites $i \neq 0$, one obtains for the direct and the exchange corrections (Fig. 2. a) and b)), respectively, the spin-isotropic terms,

$$
\begin{aligned}
\delta J^{(d)} & =-y \frac{1}{4} J g_{i}^{2} \frac{D_{0}}{\sqrt{T_{K}^{2}+T^{2}}} \frac{1}{1+\left(D / T_{K}\right)^{2}} \\
\delta J^{(e x)} & =-y \frac{1}{4} J g_{i}^{2}\left(\frac{3}{4}+\frac{T}{\sqrt{T_{K}^{2}+T^{2}}}\right) .
\end{aligned}
$$

Here $J$ is the bare, local spin exchange coupling on site $i=0$, and $g_{i}=N(0) J_{i}$ is the dimensionless, bare coupling on site $i \neq 0 . y$ is a dimensionless factor that describes the (experimentally not known) relation between the RKKY coupling strength and the $\mathrm{Au}$ content $x$. We assume a linear dependence across the QPT, $y=\alpha\left(x+x_{0}\right)$, with adjustable parameters $\alpha$ and $x_{0}$. As seen from Fig. 2 a), $\chi_{4 f}(T, \Omega)$ imposes a soft cutoff on the energy exchange in $\delta J^{(d)}$, which appears as the last factor in Eq. (1) in terms of the running band cutoff $D \leq D_{0}$. In $\delta J^{(e x)}$ (Fig. 2 b)) there is no such cutoff; however, $\chi_{4 f}(T, \Omega)$ restricts the conduction electron response to the Fermi energy, and suppresses $\delta J^{(e x)}$ compared to $\delta J^{(d)}$ by an overall factor of $\sqrt{T_{K}^{2}+T^{2}} / D_{0}$, as seen in Eq. (2). The one-loop RG equation for the local spin exchange coupling, including RKKY corrections, Eqs. (11) and (2), reads, $d J / d \ln D=-2 N(0)\left[J+\delta J^{(d)}+\delta J^{(e x)}\right]^{2}$. Note that in this RG equation the couplings $g_{i}$ on sites $i \neq 0$ are not renormalized, since this is already included in the full susceptibility $\chi_{4 f}$. The essential feature is that in the low- $T$ limit $\left(T \ll T_{K}\right)$, for which $T_{K}$ is to be extracted, the direct RKKY correction $\delta J^{(d)}$, Eq. (1), is inversely proportional to the renormalized Kondo scale $T_{K}(y)$ itself via $\chi_{4 f}(0,0) . T_{K}(y)$, including perturbative RKKY corrections, is defined as the scale where the solution of the RG equation diverges. This leads without further approximations to a highly non-linear renormalization of $T_{K}$ given by the selfconsistency equation,

$$
\frac{T_{K}(y)}{T_{K}(0)}=\exp \left\{-\left(\frac{1}{2 g}+\ln 2\right) \frac{f(u)}{1-f(u)}\right\},
$$

with $g=N(0) J, f(u)=u-u^{2} / 2, u=y g^{2} D_{0} /\left[4 T_{K}(y)\right]$. The single-ion Kondo scale without RKKY coupling is $T_{K}(0)=D_{0} \exp [-1 / 2 g]$. We have verified that this perturbative RG treatment of the RKKY corrections is controlled in the sense that the effective perturbation parameter $f(u) \leq 0.1$ for all selfconsistent solutions, i.e., the exponent in Eq. (3) remains small. The solution of Eq. (3) is universal in the dimensionless variables $T_{K}(y) / T_{K}(0)$ and $y / y_{m}$ and turns out to exist only for $y$ smaller than a maximum value $y_{m}$. For $y / y_{m} \rightarrow 1$ it behaves as $T_{K}(y) / T_{K}(0)=r+b \sqrt{1-y / y_{m}}$, with numerically determined constants $r \equiv T_{K}\left(y_{m}\right) / T_{K}(0) \approx 0.391, b \approx 0.517$. (cf. Fig. 2k)). The maximum RKKY parameter $y_{m}$ beyond which a solution of Eq. (3) ceases to exist, can be deduced in terms of $\tau_{K}=T_{K}(0) / D_{0}$ as,

$y_{m}=8 r \tau_{K}\left(\ln \tau_{K}\right)^{2}\left[2-\ln \frac{\tau_{K}}{2}-\sqrt{\left(2-\ln \frac{\tau_{K}}{2}\right)^{2}-4}\right]$ (4)

(Fig. 31 red line). For $y>y_{m}$, the Kondo spin exchange coupling including RKKY corrections does not diverge under RG. Hence, as the essential result of this analysis, in a dense system of Kondo ions, complete Kondo screening ceases to exist above a critical RKKY coupling strength, $y>y_{m}$, even if magnetic ordering does not occur. For comparison, the well-known Doniach criterion 


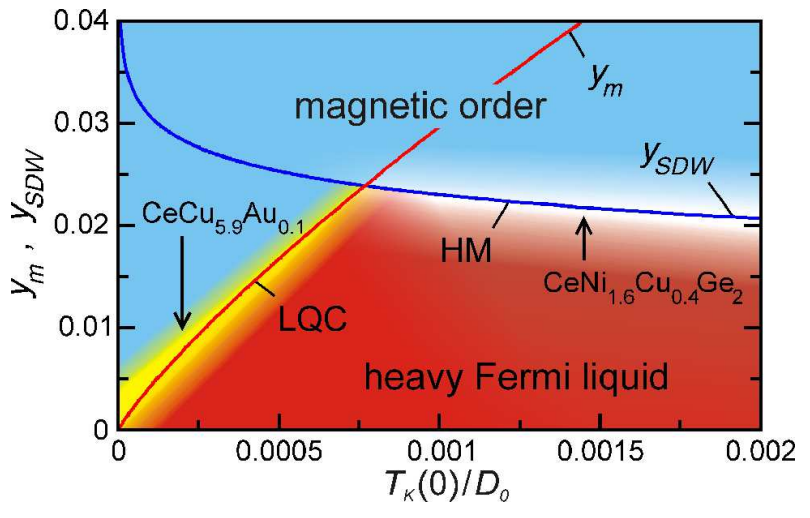

FIG. 3: (color online) Schematic $T=0$ phase diagram of a HF system with a magnetic QPT driven by RKKY coupling or a SDW instability in the plane of single-ion Kondo-scale $T_{K}(0) / D_{0}$ and RKKY coupling strength $y$, as drawn from the high- $T$ analysis. Red line $\left(y_{m}\right)$ : maximum RKKY coupling strength beyond which complete Kondo screening ceases to exist, Eq. (4). At the red line a step occurs in $T_{K}(y)$. Blue line ( $\left.y_{\text {SDW }}\right)$ : RKKY coupling strength at which the heavy Fermi liquid becomes unstable towards a SDW, assuming a spin-spin coupling $J_{S D W}=J$. The cases $y_{m}<y_{\text {SDW }}$ and $y_{m}>y_{\text {SDW }}$ distinguish whether the QPT is LQC-like (yellow) or HM-like (white), respectively. The arrows indicate estimates [16] for $T_{K}(0) / D_{0}$ for $\mathrm{CeCu}_{6-x} \mathrm{Au}_{x}$ and for $\mathrm{CeNi}_{2-x} \mathrm{Cu}_{x} \mathrm{Ge}_{2}$.

[28] pertains to the breakdown of Kondo screening due to magnetic ordering and reads $T_{K}(0) \approx y_{m} N(0) J^{2}$. It is similar, albeit not equivalent, in numerical values to Eq. (44) and does not allow for a determination of the composition-dependent screening scale $T_{K}(y)$. By identifying $y_{m}$ with the QCP, as done in Fig. 2 the $x$ dependence of $T_{K}[y(x)]$ near this critical endpoint explains the abrupt step of $T_{K}$ observed experimentally in the high$T$ spectral data. The small KR spectral weight seen for $x>x_{c}$ should be interpreted merely as the logarithmic onset of Kondo screening which does not fully develop even for $T \rightarrow 0$. Consequently, the small, residual local moments must order in dimensions $d>2$ at sufficiently low $T$.

Hence, the theory predicts two possible scenarios, depending on the size of $T_{K}(0) / D_{0}$ : (1) Magnetic ordering at $T=0$ occurs for an RKKY parameter $y=y_{\mathrm{SDW}}<y_{m}$, i.e., without breakdown of Kondo screening. The ordering may arise in this case from a $T=0$ spin-density-wave (SDW) instability of the underlying heavy Fermi liquid. This corresponds to the HM scenario, depicted in Fig. 3 as the white region. In this case, a step-like behavior of $T_{K}$ as extracted from the high- $T$ UPS spectra is still predicted from Eq. (3) at $y=y_{m}$, i.e., inside the region where magnetic ordering occurs at $T<T_{N}$. (2) Magnetic ordering does not occur for $y<y_{m}$. In this case Eq. (3) indicates a breakdown of Kondo screening at the magnetic QCP, and quantum critical fluctuations (not considered in our theory) will suppress the low- $T$ spin screening scale to zero at this point. This is the LQC scenario, shown in Fig. 3 as the yellow region.
To conclude, the theoretical analysis predicts generally that an abrupt step of the Kondo screening scale extracted from high- $T$ spectral data should occur in any HF compound with competing Kondo and RKKY interactions, as long as the single-ion Kondo screening scale is larger than the magnetic ordering temperature. Whether this distinct feature is located at the quantum critical control parameter value $x_{c}$ or inside the magnetically ordered region constitutes a general high- $T$ criterion to distinguish the LQC and HM scenarios. The sharp step of $T_{K}$ occurring in our UPS data very close to $x_{c}$ (see Fig. 2) strongly supports that $\mathrm{CeCu}_{6-x} \mathrm{Au}_{x}$ falls into this class, as was previously inferred indirectly from inelastic neutron scattering data [14].

We would like to thank F. Assaad, S. Kirchner, and M. Vojta for fruitful discussions. This work was supported by DFG through Re 1469/4-3/4 (M.K., A.N., F.R.), SFB 608 (J.K.) and FOR 960 (H.v.L.).

[1] H. v. Löhneysen, A. Rosch, M. Vojta, and P. Wölfle, Rev. Mod. Phys. 79, 1015 (2007).

[2] P. Gegenwart, Q. Si, and F. Steglich, Nature Phys. 4, 186 (2008).

[3] J. A. Hertz, Phys. Rev. B 14, 1165 (1976).

[4] A. J. Millis, Phys. Rev. B 48, 7183 (1993).

[5] Q. Si, S. Rabello, and J. L. Smith, Nature 413, 804 (2001).

[6] P. Coleman, C. Pépin, Q. Si, and R. Ramazashvili, J. Phys.: Condens. Matter 13, R723 (2001).

[7] H. v. Löhneysen et al., Phys. Rev. Lett. 72,3262 (1994).

[8] H. v. Löhneysen, J. Phys. Cond. Mat. 8, 9689 (1996).

[9] H. v. Löhneysen et al., J. Magn. Mag. Mat. 177-181, 12 (1998).

[10] O. Stockert et al., Phys. Rev. Lett. 80,5627 (1998).

[11] H. v. Löhneysen et al., Eur. Phys. J. B 5, 447 (1998).

[12] B. Stroka et al., Z. Phys. B 90, 155 (1993).

[13] K. Grube et al., Phys. Rev. B 60, 11947 (1999).

[14] A. Schröder et al., Nature 407, 351 (2000).

[15] F. Reinert et al., Phys. Rev. Lett. 87, 106401 (2001).

[16] D. Ehm et al., Phys. Rev. B 76, 045117 (2007).

[17] M. Klein et al., Phys. Rev. Lett. 101, 046406 (2008).

[18] J. Kroha et al., Physica E 18, 69 (2003).

[19] H. v. Löhneysen et al., J. Alloys Compd. 408-412, 9 (2006).

[20] H. G. Schlager, A. Schröder, M. Welsch, and H. v. Löhneysen, J. Low Temp. Phys. 90, 181 (1993).

[21] F. Patthey et al., Phys. Rev. B 42, 8864 (1990).

[22] M. Garnier et al., Phys. Rev. Lett. 78, 4127 (1997).

[23] J. Allen et al., et al., J. Appl. Phys. 87, 6088 (2000).

[24] D. Ehm et al., Acta Phys. Pol. B 34, 951 (2003).

[25] T. Costi, J. Kroha, and P.Wölfle, Phys. Rev. B 53, 1850 (1996); N. E. Bickers, D. L. Cox, and J. W. Wilkins, Phys. Rev. B 36, 2036 (1987).

[26] B. A. Jones and C. M. Varma, Phys. Rev. Lett. 58, 843 (1987).

[27] H. Maebashi, K. Miyake, and C. M. Varma, Phys. Rev. Lett. 95, 207207 (2005).

[28] S. Doniach, Physica B \& C 91, 231 (1977). 